\title{
Intraspecific variation in the strength of density dependence in aphid populations
}

\author{
ANURAG A. AGRAWAL, ${ }^{1}$ NORA UNDERWOOD ${ }^{2}$ and JOHN \\ R. S T I N C H C O M B E ${ }^{3}{ }^{1}$ Department of Botany, University of Toronto, Canada, ${ }^{2}$ Department of Biological \\ Science, Florida State University, U.S.A., and ${ }^{3}$ Department of Ecology and Evolutionary Biology, Brown University, \\ Providence, Rhode Island, U.S.A.
}

\begin{abstract}
Experimental evidence is presented for positive, negative, and no density dependence from 32 independent density manipulations of milkweed aphids (Aphis nerii) in laboratory and field experiments. This substantial variation in intraspecific density dependence is associated with temperature and host-plant species.

2. It is reported that as population growth rate increases, density dependence becomes more strongly negative, suggesting that the monotonic definition of density dependence used in many common population models is appropriate for these aphids, and that population growth rate and carrying capacity are not directly proportional.

3. For populations that conform to these assumptions, population growth rate may be widely applicable as a predictor of the strength of density dependence.
\end{abstract}

Key words. Aphis nerii, Asclepias syriaca, density dependence, density manipulation experiment, logistic population growth, milkweed, plant-herbivore interactions, population regulation.

\section{Introduction}

Density dependence in mortality and reproduction has been demonstrated in both time-series analyses and manipulative experiments, and the strength of density dependence varies both within and between studies (Strong, 1984; Bjornstad et al., 1995; Cappuccino \& Price, 1995; Harrison \& Cappuccino, 1995; Wolda, 1995; Krebs, 2002). However, relatively little experimental data address the conditions that may promote strong vs. weak density dependence in a particular species. To incorporate density dependence into a predictive framework for population dynamics, the magnitude, causes, and correlates of variation in the strength of density dependence will need to be understood. In this study, it was sought to characterise variation in density dependence in an easily manipulated plant-insect system and to consider factors that might influence that variation.

One factor that is expected to be related to the strength of density dependence (i.e. slope of the relationship between

Correspondence: Anurag A. Agrawal, Department of Ecology and Evolutionary Biology, Corson Hall, Cornell University, Ithaca, NY 14853, U.S.A. E-mail: anurag.agrawal@utoronto.ca density and per capita growth rate) is population growth rate at low density (i.e. what is typically $R_{\max }$ ). This expectation is based on the monotonic definition of density dependence that is included in the most widely used singlespecies models of population dynamics [e.g. the logistic (Pearl \& Reed, 1920), Ricker (Ricker, 1954), Hassell (Hassell, 1975), and Beverton-Holt (Beverton \& Holt, 1993) models]. For example, in the logistic model

$$
\frac{\mathrm{d} N}{\mathrm{~d} t}=r N\left(1-\frac{N}{K}\right),
$$

where $N$ is the population density, $t$ is time, $r$ is the maximum population growth rate, and $K$ is the carrying capacity, density dependence is defined as a linear relationship between density and per capita population growth rate,

$$
\frac{\mathrm{d} N}{\mathrm{~d} t N}=r-\frac{r}{K} N
$$

The slope of this relationship (i.e. the strength of density dependence) is equal to $-(r / K)$. Therefore, for populations that conform to the logistic model or other models using a monotonic definition of density dependence, as long as 
changes in growth rate do not cause directly proportional changes in carrying capacity, increases in growth rate should by definition be associated with increases in the strength of density dependence (Fig. 1). Commonly employed consumer resource models make the same prediction (MacArthur, 1970; Abrams, 1998), although $r$ and $K$ are defined in terms of resource renewal and consumer functional response. The presence of population growth rate in the definition of density dependence in these models is well known, yet empirical studies have rarely considered the relationship between $r$ and the strength of density dependence (but see Krüger et al., 2002 for an exception). For systems that conform to the common monotonic definition of density dependence, the expected relationship between $r$ and the strength of density dependence could be used to predict or explain variation in the strength of density dependence.

In this study, the effects of varying densities of Aphis nerii on individual milkweed plants (Asclepias spp.) were examined to assess variation in per capita growth rates in 32 independent experiments. A decrease in per capita population growth as initial density increases corresponds to negative density dependence. The analysis of density dependence follows from standard definitions and models (Gotelli, 2001) and this manipulative approach has been successfully employed for estimating the strength of density dependence (Hunter \& Elkinton, 1999; Underwood \& Rausher, 2000; Underwood \& Rausher, 2002; Rotem \& Agrawal, 2003). Using these data on density dependence under different

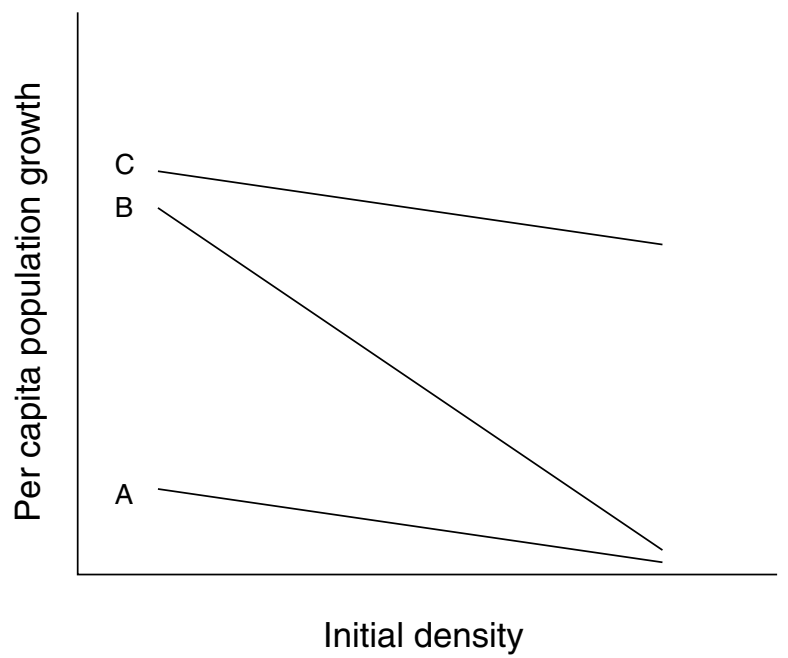

Fig. 1. A hypothetical depiction of density dependence in three populations. The $Y$ intercept represents the maximum population growth rate $(r)$ of a population and $X$ intercept represents the carrying capacity $(K)$. Where $K$ remains constant, or at least does not scale directly proportionately with $r$ (populations A and B), populations with a greater $r$ will have steeper negative slopes of density dependence. However, if $r$ and $K$ scale directly proportionately (populations A and C) the slope of density dependence remains constant. conditions in one species, considerable intraspecific variation in the strength of density dependence is demonstrated, several factors that influence the strength of density dependence are noted, and whether variation in the maximum population growth rate predicts the strength of density dependence observed is considered.

\section{Materials and methods}

Aphis nerii is a cosmopolitan aphid species that typically reproduces by parthenogenesis; it has a limited host range and feeds on Nerium spp. and plants in the Ascelpiadaceae (oleander and milkweeds), from which it sequesters toxic cardenolides (cardiac glycosides) (Rothschild et al., 1970). Nonetheless, plant cardenolides negatively correlate with the population growth rate of aphids (Agrawal, 2004). Individuals typically grow in clusters, and the progeny of a single adult are especially aggregated (Hall \& Ehler, 1980). Although individuals are not very mobile and do not usually walk off a suitable host plant, under dense conditions winged dispersal morphs are produced (Groeters, 1989).

The experiments in the work reported here fall into four categories: (A) laboratory manipulations of aphid densities on potted milkweed (Asclepias syriaca) plants, (B) field manipulations of aphid densities on single A. syriaca plants in natural milkweed populations, (C) laboratory manipulations of aphid densities and temperature (a densityindependent factor affecting population growth rates) on A.syriaca, and (D) laboratory manipulations of aphid densities on various other species of Asclepias spp.

In all experiments, the density of aphids on individual plants was manipulated by placing two adult gravid aphids near the apex of each plant. After 2 days, the adults were removed and the offspring were thinned to the randomly assigned number of aphids. Plants were checked the following day and any minor adjustments to meet the assigned initial aphid densities were made. In the laboratory experiments (A), initial density ranged from 1 to 20 aphids per plant; sample sizes were between 10 and 45 plants per experiment; a total of eight independent experiments were conducted (see Table 1 for full details). Milkweed plants were grown in 500-ml pots in growth chambers. Plants were grown for approximately 1 month (until they had 10-16 leaves) before use in experiments.

In the field experiments (B), initial density ranged from 1 to 30 aphids per plant; sample sizes were 30 plants per experiment. Two independent experiments were conducted, one in the spring (May) and one in the summer (August). Field experiments were conducted in southern Ontario (Koffler Scientific Reserve at Jokers Hill, $44^{\circ} 03^{\prime} \mathrm{N}, 79^{\circ} 29^{\prime} \mathrm{W}$, http:// www.zoo.utoronto.ca/jokershill). Aphis nerii can be abundant on milkweed during the summer. In natural populations of plants, 30 stems at least $1 \mathrm{~m}$ away from each other were selected and aphids were placed on their apices.

For the laboratory experiments (C) with temperature manipulations, initial density ranged from 1 to 15 aphids per plant; sample sizes ranged from 18 to 20 plants per 
Table 1. Summary of the first 12 independent experiments of Aphis nerii on Asclepias syriaca; 20 other experiments are reported in Agrawal (2004). Categories are as follows: (A) laboratory manipulations of aphid densities on single potted plants, (B) field manipulations of aphid densities on single plants in natural milkweed populations, and (C) laboratory manipulations of aphid density and temperature. Temperatures are given as day/night temperatures.

\begin{tabular}{|c|c|c|c|c|c|c|c|c|}
\hline Experiment & Category & Initial density range & Temperature $\left({ }^{\circ} \mathrm{C}\right)$ & Sample size & Intercept & Slope & $r^{2}$ & $P$ \\
\hline 1 & A & $2-20$ & $28 / 24$ & 10 & 0.629 & -0.012 & 0.920 & $<0.001$ \\
\hline 2 & A & $2-20$ & $28 / 24$ & 10 & 0.600 & -0.009 & 0.763 & $<0.001$ \\
\hline 3 & $\mathrm{~A}$ & $2-20$ & $28 / 24$ & 10 & 0.549 & -0.010 & 0.794 & $<0.001$ \\
\hline 4 & A & $1-12$ & $22 / 22 *$ & 34 & 0.397 & 0.002 & 0.009 & 0.599 \\
\hline 5 & A & $1-8$ & $25 / 20^{*}$ & 16 & 0.346 & -0.005 & 0.014 & 0.657 \\
\hline 6 & $\mathrm{~A}$ & $1-15$ & $28 / 24$ & 22 & 0.551 & -0.001 & 0.025 & 0.483 \\
\hline 7 & A & $1-15$ & $28 / 24$ & 19 & 0.440 & -0.005 & 0.104 & 0.178 \\
\hline 8 & A & $1-15$ & $28 / 24$ & 45 & 0.641 & -0.009 & 0.383 & $<0.001$ \\
\hline 9 & $\mathrm{~B}$ & $1-30$ & $18 / 12^{*}$ & 25 & -0.020 & 0.008 & 0.254 & 0.010 \\
\hline 10 & $\mathrm{~B}$ & $1-30$ & $28 / 24^{*}$ & 30 & 0.248 & -0.008 & 0.111 & 0.072 \\
\hline 11 & $\mathrm{C}$ & $1-15$ & $22 / 18$ & 20 & 0.267 & -0.002 & 0.119 & 0.136 \\
\hline 12 & $\mathrm{C}$ & $1-15$ & $28 / 24$ & 18 & 0.401 & -0.010 & 0.832 & $<0.001$ \\
\hline
\end{tabular}

*Estimated temperature.

experiment. A total of two independent experiments were conducted where plants were grown in either warm or cool environments: one growth chamber (cool) was kept at $22^{\circ} \mathrm{C}$ (day) and $18{ }^{\circ} \mathrm{C}$ (night), while the other (warm) was kept at $28^{\circ} \mathrm{C}$ (day) and $24^{\circ} \mathrm{C}$ (night). The chambers are identical in make, model, and age and were set on a LD 14:10 h cycle. To account for potential effects associated with the two chambers, the temperature programmes and the plants were switched midway through the experiment.

In the final set of experiments (D), the initial density of aphids was manipulated ranging from 1 to 15 aphids on each of 18 species of Asclepias. Experimental procedures were essentially identical to the growth chamber experiments described above. The experiments were conducted in a common growth chamber environment with replicates of plant species completely randomised. These data, representing 20 independent experiments, are presented in a separate manuscript, assessing the role of plant defensive traits in aphid population dynamics (Agrawal, 2004).

\section{Data collection and analysis}

In all cases, aphid populations were sampled once after a week of growth, representing roughly two full generations at warm temperatures. Aphids were counted exhaustively on the stems and tops and bottoms of all leaves. The strength of density dependence was measured as the slope of the regression of daily per capita population growth rate vs. initial aphid density (Gotelli, 2001). Per capita growth rate was defined by the logistic model, where per capita growth rate $(\mathrm{d} N / \mathrm{d} t N)$ was estimated as

$$
\frac{\left(\ln N_{2}-\ln N_{1}\right)}{t_{2}-t_{1}} \text {. }
$$

The population growth rate at low density $\left(R_{\text {int }}\right)$ was estimated as the $Y$ intercept of these regressions - that is, the expected population growth rate of aphids as density approached zero. For cases where density dependence is negative, $R_{\text {int }}$ is the maximum possible growth rate. Although the strength of density dependence may be overestimated by regressing the rates of population growth on population density in a time series, this manipulative approach is a statistically sound method for estimating effects of density (Prairie \& Bird, 1989; Cappuccino, 1992; Griffiths, 1998).

The hypothesis that the strength of density dependence is correlated with the population growth rate at low density $\left(R_{\text {int }}\right)$ was tested. Operationally, this corresponds to evaluating the statistical significance of the correlation or covariance between the slopes and intercepts estimated for the 32 experiments. However, because the slope and intercept of an individual experiment are estimated from the same data, an artefactual covariance exists between these two estimates that inflates the estimated covariance. To address this problem, statistical methods from the ecological genetics literature (e.g. Appendix B of Mauricio et al., 1997 and Appendix B of Tiffin \& Rausher, 1999) were used to calculate and remove the artefactual covariance between $R_{\text {int }}$ and the strength of density dependence. To test the statistical significance of the corrected covariance between $R_{\text {int }}$ and the strength of density dependence, it was determined whether the $99 \%$ confidence limits for the corrected covariance, calculated from 1000 bootstraps, overlapped zero.

\section{Results}

In eight laboratory experiments (category A in Table 1), the average per capita aphid population growth was very high (mean per capita growth per day $\pm \mathrm{SE}=0.478 \pm 0.008$ ), and experimentally varying the density of aphids generally resulted in negative density dependence (Table 2, Fig. 2). However, the strength of density dependence varied considerably from trial to trial, ranging from 0.008 to 0.012 
Table 2. The influence of initial aphid density and trial (category A from Table 1) on per capita growth rate of the population over about two complete generations. $r^{2}=0.734$. The significant interaction between aphid density and trial indicates that the slopes of density dependence varied from trial to trial.

\begin{tabular}{lrlll}
\hline Source & d.f. & Mean-square & \multicolumn{1}{l}{$F$} & \multicolumn{1}{l}{$P$} \\
\hline Aphid density & 1 & 0.080 & 25.363 & $<0.001$ \\
Trial & 7 & 0.059 & 18.610 & $<0.001$ \\
Aphid density $\times$ Trial & 7 & 0.009 & 2.824 & 0.009 \\
Error & 150 & 0.003 & & \\
\hline
\end{tabular}

(Fig. 2, interaction term in Table 2). In the spring field experiment, average daily per capita aphid population growth was low $(0.05 \pm 0.02)$, and experimentally varying the density of aphids resulted in significantly positive density dependence (Table 1, experiment 9). In the summer field experiment, aphids had relatively high daily per capita growth $(0.11 \pm 0.04)$ and populations experienced negative density dependence (Table 1 , experiment 10).

Because the above-described experiments provided evidence for substantial variance in density dependence, additional experiments were conducted to address factors that might affect the strength of density dependence in aphid populations. Growth rate independent of density was manipulated by imposing warm and cold treatments. Low growth rates at lower temperatures did not result in density dependence (Table 1, experiment 11), whereas at higher temperatures, density dependence was strongly negative (Table 1, experiment 12). In a separate analysis of the temperature manipulation trials, a significant temperature by initial density interaction was detected $\left(F_{1,34}=15.363\right.$, $P<0.001)$. When density manipulations were conducted on 18 different species of host plant, considerable variation in the strength of density dependence was also found (plant

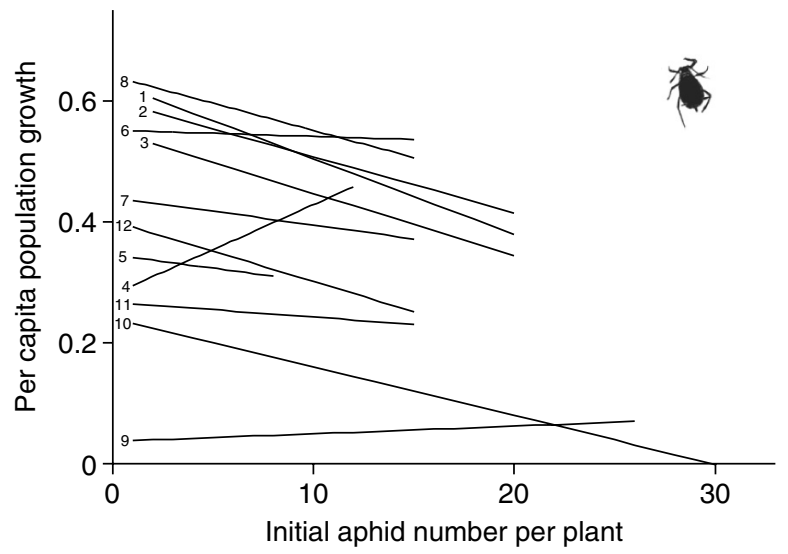

Fig. 2. Effects of manipulation of aphid density on per capita growth rates on milkweed plants in 12 laboratory and field experiments. Each line represents an independent density manipulation experiment (data points are not shown as they obscure the clarity of the graph). The identity of the experimental trials corresponds to Table 1 and is indicated to the left of each line. species by initial density interaction: $F_{17,253}=1.804$, $P=0.028)$ (Agrawal, 2004)

In a single analysis, employing each of the 32 independent experiments as single replicates, it was found that $R_{\text {int }}$ was strongly negatively correlated with the slope of density dependence (Fig. 3, $r=-0.73, P<0.001$; corresponding to a covariance of -0.0006$)$. Of this estimated covariance between $R_{\text {int }}$ and the strength of density dependence, approximately $12.4 \%(-0.00007)$ was due to the artefactual covariance introduced by estimating $R_{\text {int }}$ and the strength of density dependence from the same data in each experimental trial. The corrected covariance $(-0.0005)$ is significantly different from zero (99\% confidence limits $=-0.0002,-0.0006)$, suggesting that aphid populations with higher population growth rates at low density were subject to stronger density dependence (Fig. 3).

\section{Discussion}

It has been found that density dependence varies substantially within an interaction between aphid herbivores and their hosts, and that the sign and strength of the density dependence were affected by conditions such as temperature and species of host plant. These results from aphid populations are consistent with previous reports of intraspecific variation in density dependence from other taxonomic groups (Hunter \& Elkinton, 1999; Underwood \& Rausher, 2000, 2002; Krebs, 2002; Krüger et al., 2002; Rotem \& Agrawal, 2003).

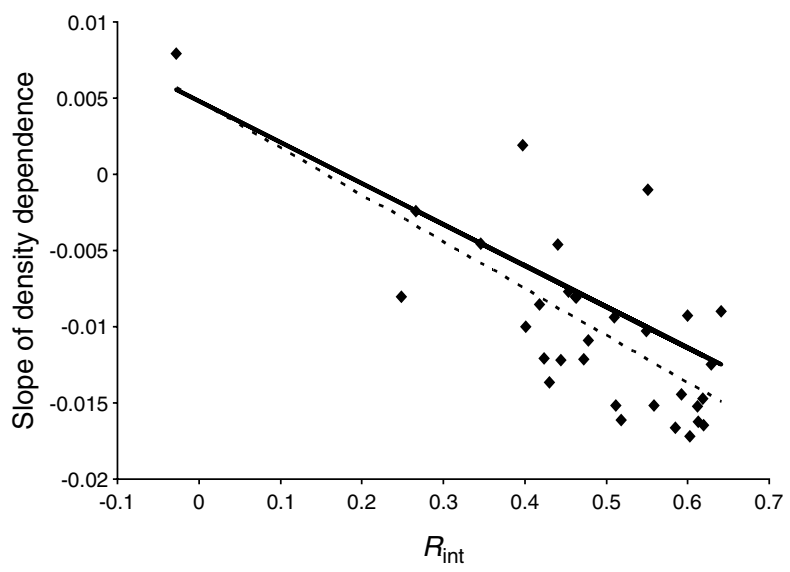

Fig. 3. The strength of density dependence across 32 independent experiments is predicted by the population growth rate $\left(R_{\text {int }}\right)$ from each experiment. Each data point represents the slope and $Y$ intercept from a regression of per capita growth rates on initial density. For heuristic purposes, the corrected covariance is portrayed as a regression line (solid line), calculated with the same intercept and variance in the independent variable as would be obtained from a simple regression with uncorrected data (dashed line). Accordingly, the covariance between $X$ and $Y$ is the only term that differs between the regression lines. 
After correcting for artefactual covariance, it was found that the strength of density dependence in aphid populations was strongly correlated with population growth rate at low density $\left(R_{\text {int }}\right)$, consistent with the monotonic definition of density dependence assumed in many commonly used population models. This result was found across experiments performed at different times and locations, regardless of whether variation in the strength of density dependence was influenced by abiotic conditions (temperature) or resource quality (host-plant species). Krüger et al. (2002) noted a similar negative relationship between population growth rate and strength of density dependence in observational data from 15 South African raptor species. While a relationship between growth rate and strength of density dependence is known to be inherent in the definition of density dependence models such as the logistic and Ricker, this relationship has not been widely examined empirically. This study suggests that the monotonic definition of density dependence is applicable to this aphid species.

To the extent that systems in general conform to the monotonic definition of density dependence, the relationship between growth rate and the strength of density dependence may be useful in predicting variation in density dependence across sites, years, and species. For example, when organisms live along environmental gradients associated with temperature or resource availability, such as altitude and latitude, populations should experience greater strength of density dependence as growth rates change along the gradient if those organisms conform to the monotonic definition of density dependence (Bjornstad et al., 1995). The recent global discovery that plants show facilitation (or positive effects) at harsher, high altitudes, and competition at low, benign altitudes (Callaway et al., 2002), is consistent with the hypothesis that factors influencing population growth rate may influence the sign and strength of density dependence.

A relationship between growth rate and density dependence is only predicted from single species models if population growth rate and carrying capacity are not directly proportional (although they may still be correlated) (Fig. 1). Thus, results of this study and of Krüger et al. (2002) suggest that growth rate and carrying capacity do not scale directly proportionally. Although the relationship between $r$ and $K$ has not been widely explored empirically, the relationship between these two key parameters of population growth (or factors that influence them) bares on a wide variety of topics in population biology. For example, a substantial evolutionary literature ( $r-K$ selection theory) considers possible negative correlations between $r$ and $K$ (Mueller, 1997). Bell (1991) reported positive and negative relationships between $r$ and $K$ for Chlamydomonas, depending on the nutrient environment. In a very different area, some population viability analyses that employ mean variances to predict changes in populations implicitly assume proportional change in $r$ and $K$, because an environmental or demographic effect on per capita growth is assumed to have the same consequence at high and low densities (Morris \& Doak, 2002).
Many hypotheses in ecology and evolution test for the relationship between two parameters that are estimated from the same data set. In this study, the relationship between the slopes and intercepts of common regressions was examined involving per capita population growth rate vs. initial density. In the ecological genetics literature, similar relationships have been used to examine fitness costs of plant tolerance to herbivory (Mauricio et al., 1997; Tiffin \& Rausher, 1999). The use of statistical tools from the ecological genetics literature - which may have wide application in biology - is indicative of a more general phenomenon than the statistical correction itself: the substantial overlap in analytical and conceptual problems addressed by population and community ecologists and ecological and community geneticists [see Fukami \& Morin (2003) for examples]. Given the high level of variation in population growth rates of a species in space and time, this statistically unbiased analysis reveals a previously under-appreciated, yet predictable relationship with the strength of density dependence.

\section{Acknowledgements}

We thank Peter Abrams, Chad Brassil, John Fryxell, Mark Hunter, Brian Inouye, Marc Johnson, Rick Karban, Nile Kurashige, Lisa Plane, Karin Rotem, Jennifer Thaler, and Peter Van Zandt for help with the experiments, discussion, or comments on the manuscript. A.A.A.'s research and laboratory (http://www.herbivory.com) are supported by the NSERC of Canada, the Canadian Foundation for Innovation, and a Premier's Research Excellence Award from the Ontario government; N.U. was supported by the US NSF; J.R.S. was supported by US NSF grant DEB-0129018 to Johanna Schmitt. A macro written in SAS that performs the covariance correction and bootstrapping is available from J.R.S.

\section{References}

Abrams, P.A. (1998) High competition with low similarity and low competition with high similarity: exploitative and apparent competition in consumer-resource systems. American Naturalist, 152, 114-128.

Agrawal, A.A. (2004) Plant defense and density dependence in the population growth of herbivores. American Naturalist, 164, 113-120.

Bell, G. (1991) The ecology and genetics of fitness in Chlamydomonas III. Genotype-by-environment interaction within strains. Evolution, 45, 668-679.

Beverton, R.J.H. \& Holt, S. (1993) The Dynamics of Exploited Fish Populations. Chapman \& Hall, London.

Bjornstad, O.N., Falck, W. \& Stenseth, N.C. (1995) A geographic gradient in small rodent density fluctuations: a statistical modelling approach. Proceedings of the Royal Society of London, Series B, 262, 127-133.

Callaway, R.M., Brooker, R.W., Choler, P., Kikvidze, Z., Lortie, C.J., Michalet, R. et al. (2002) Positive interactions among alpine plants increase with stress. Nature, 417, 844-848. 
Cappuccino, N. (1992) The nature of population stability in Eurosta solidaginis, a nonoutbreaking herbivore of goldenrod. Ecology, 73, 1792-1801.

Cappuccino, N. \& Price, P.W., eds (1995) Population Dynamics. Academic Press, San Diego.

Fukami, T. \& Morin, P.J. (2003) Productivity-biodiversity relationships depend on the history of community assembly. Nature, 424, 423-426.

Gotelli, N.J. (2001) A Primer of Ecology, 3rd edn. Sinauer, Sunderland, Massachusetts.

Griffiths, D. (1998) Sampling effort, regression method, and the shape and slope of size-abundance relations. Journal of Animal Ecology, 67, 795-804.

Groeters, F.R. (1989) Geographic and clonal variation in the milkweed-oleander aphid, Aphis nerii (Homoptera, Aphididae), for winged morph production, life-history, and morphology in relation to host plant permanence. Evolutionary Ecology, 3, 327-341.

Hall, R.W. \& Ehler, L.E. (1980) Population ecology of Aphis nerii Homoptera, Aphididae on oleander. Environmental Entomology, 9, 338-344.

Harrison, S. \& Cappuccino, N. (1995) Using density manipulation experiments to study population regulation. Population Dynamics (ed. by N. Cappuccino and P. Price), pp. 131-147. Academic Press, San Diego, California.

Hassell, M.P. (1975) Density-dependence in single-species populations. Journal of Animal Ecology, 44, 283-295.

Hunter, A.F. \& Elkinton, J.S. (1999) Interaction between phenology and density effects on mortality from natural enemies. Journal of Animal Ecology, 68, 1093-1100.

Krebs, C.J. (2002) Two complementary paradigms for analysing population dynamics. Philosophical Transactions of the Royal Society of London. Series B - Biological Sciences, 357, 1211-1219.

Krüger, O., Liversidge, R. \& Lindström, J. (2002) Statistical modelling of the population dynamics of a raptor community in a semi-desert environment. Journal of Animal Ecology, 71, 603-613.

MacArthur, R.H. (1970) Species packing and competitive equilibria for many species. Theoretical Population Biology, 1, 1-11.

Mauricio, R., Rausher, M.D. \& Burdick, D.S. (1997) Variation in the defense strategies of plants: are resistance and tolerance mutually exclusive? Ecology, 78, 1301-1311.
Morris, W.F. \& Doak, D.F. (2002) Quantitative Conservation Biology: Theory and Practice of Population Viability Analysis. Sinauer Associates, Sunderland.

Mueller, L.D. (1997) Theoretical and empirical examination of density-dependent selection. Annual Review of Ecology and Systematics, 28, 269-288.

Pearl, R. \& Reed, L.J. (1920) On the rate of growth of the population of the United States since 1790 and its mathematical representation. Proceedings of the National Academy of Sciences of the United States of America, 6, 275-288.

Prairie, Y.T. \& Bird, D.F. (1989) Some misconceptions about the spurious correlation problem in the ecological literature. Oecologia, 81, 285-288.

Ricker, W.E. (1954) Stock and recruitment. Journal of the Fisheries Research Board of Canada, 11, 559-623.

Rotem, K.A. \& Agrawal, A.A. (2003) Density dependent population growth of the two-spotted spider mite, Tetranychus urticae, on the host plant Leonurus cardiaca. Oikos, 103, 559-565.

Rothschild, M., Euw, J.V. \& Reichste, T. (1970) Cardiac glycosides in oleander aphid, Aphis nerii. Journal of Insect Physiology, 16, $1141-1145$.

Strong, D.R. (1984) Density-vague ecology and liberal population regulation in insects. A New Ecology: Novel Approaches to Interactive Systems (ed. by P. W. Price, C. N. Slobodchikoff and W. S. Gaud), pp. 313-327. Wiley, New York.

Tiffin, P. \& Rausher, M.D. (1999) Genetic constraints and selection acting on tolerance to herbivory in the common morning glory Ipomoea purpurea. American Naturalist, 154, 700-716.

Underwood, N. \& Rausher, M.D. (2000) The effects of host-plant genotype on herbivore population dynamics. Ecology, 81, $1565-1576$.

Underwood, N. \& Rausher, M.D. (2002) Comparing the consequences of induced and constitutive plant resistance for herbivore population dynamics. American Naturalist, 160 , 20-30.

Wolda, H. (1995) The demise of the population regulation controversy. Researches on Population Ecology, 37, 91-93.

Accepted 3 February 2004 